\title{
High Serum Adipocyte Fatty Acid Binding Protein Is Associated with Metabolic Syndrome in Patients with Type 2 Diabetes
}

\author{
Jer-Chuan Li, ${ }^{1}$ Du-An Wu, ${ }^{1,2}$ Jia-Sian Hou, ${ }^{3,4}$ Yi-Maun Subeq, \\ Hsin-Dean Chen, ${ }^{1}$ and Bang-Gee $\mathrm{Hsu}^{2,3,4}$ \\ ${ }^{1}$ Division of Metabolism and Endocrinology, Buddhist Tzu Chi General Hospital, Hualien, Taiwan \\ ${ }^{2}$ School of Medicine, Tzu Chi University, Hualien, Taiwan \\ ${ }^{3}$ Division of Nephrology, Buddhist Tzu Chi General Hospital, Hualien, Taiwan \\ ${ }^{4}$ Institute of Medical Sciences, Tzu Chi University, Hualien, Taiwan \\ ${ }^{5}$ Department of Nursing, Tzu Chi University, Hualien, Taiwan
}

Correspondence should be addressed to Bang-Gee Hsu; gee.lily@msa.hinet.net

Received 12 September 2016; Revised 31 October 2016; Accepted 9 November 2016

Academic Editor: Osama M. Ahmed

Copyright (C) 2016 Jer-Chuan Li et al. This is an open access article distributed under the Creative Commons Attribution License, which permits unrestricted use, distribution, and reproduction in any medium, provided the original work is properly cited.

\begin{abstract}
Adipocyte fatty acid binding protein (A-FABP) is a key mediator of obesity-related metabolic syndrome (MetS). The aim of this study was to evaluate the relationship between A-FABP concentration and MetS in type 2 diabetes mellitus (DM) patients. Fasting blood samples were obtained from 165 type 2 DM volunteers. MetS and its components were defined using diagnostic criteria from the International Diabetes Federation. Among 165 DM patients, 113 patients (68.5\%) had MetS. Diabetic persons who had MetS had significantly higher A-FABP levels $(P<0.001)$ than those without MetS. Female DM persons had higher A-FABP level than man $(P<0.001)$. No statistically significant differences in A-FABP levels were found in use of statin, fibrate, or antidiabetic drugs. Multivariate forward stepwise linear regression analysis revealed that body fat mass $(P<0.001)$, logarithmically transformed creatinine (log-creatinine; $P<0.001)$, female DM patients $(P<0.001)$, and logarithmically transformed high sensitive C-reactive protein (log-hs-CRP; $P=0.013)$ were positively correlated, while albumin $(P=0.004)$ and glomerular filtration rate $(\mathrm{GFR} ; P=$ 0.043 ) were negatively correlated with serum A-FABP levels in type $2 \mathrm{DM}$ patients. In this study, higher serum A-FABP level was positively associated with MetS in type $2 \mathrm{DM}$ patients.
\end{abstract}

\section{Introduction}

Metabolic syndrome (MetS) is a clustering of metabolic risk factors for atherosclerotic cardiovascular disease and diabetes mellitus (DM) [1]. There are more than 415 million people affected by DM worldwide and it is one of the most important public health challenges of the twenty-first century [2].

Adipocyte fatty acid binding protein (A-FABP) is one of the most abundant proteins in mature adipocytes, which is known for the ability to bind fatty acids and related compounds throughout various cellular compartments, including the peroxisomes, mitochondria, endoplasmic reticulum, lipid droplets, and nucleus [3, 4]. A-FABP has been shown to affect insulin sensitivity, lipid metabolism, and lipolysis in animal studies [3]. Furthermore, studies also found that AFABP is a key mediator for the obesity-related cardiovascular disease and is positively associated with MetS [3, 5]. Our previous studies noted that A-FABP was associated with MetS in coronary artery disease and in hemodialysis patients $[6,7]$. The aim of this study was to investigate the relationship between fasting serum A-FABP level and the MetS among type 2 DM patients.

\section{Materials and Methods}

2.1. Patients. There were 165 type 2 DM patients enrolled from a medical center in Hualien, Taiwan, from November 2014 through March 2015. The Protection of the Human Subjects Institutional Review Board of Tzu Chi University and Hospital approved this study. All patients provided their informed consents before participating in this study. Blood pressure (BP) was measured by trained staff in the 
TABLE 1: Clinical variables of the 165 diabetic patients with or without metabolic syndrome.

\begin{tabular}{|c|c|c|c|c|}
\hline Items & $\begin{array}{l}\text { All participants } \\
\quad(n=165) \\
\end{array}$ & $\begin{array}{l}\text { No metabolic syndrome } \\
\qquad(n=52)\end{array}$ & $\begin{array}{c}\text { Metabolic syndrome } \\
\quad(n=113) \\
\end{array}$ & $P$ value \\
\hline Age (years) & $65.00(57.00-70.00)$ & $62.00(56.25-67.00)$ & $66.00(57.50-71.00)$ & 0.159 \\
\hline Height $(\mathrm{cm})$ & $161.75 \pm 8.41$ & $163.20 \pm 7.86$ & $161.08 \pm 8.61$ & 0.132 \\
\hline Body weight (kg) & $70.69 \pm 13.36$ & $63.09 \pm 8.88$ & $74.19 \pm 13.65$ & $<0.001^{*}$ \\
\hline Body mass index $\left(\mathrm{kg} / \mathrm{m}^{2}\right)$ & $26.91 \pm 3.94$ & $23.62 \pm 2.30$ & $28.43 \pm 3.60$ & $<0.001^{*}$ \\
\hline Body fat mass (\%) & $31.49 \pm 7.55$ & $23.36 \pm 5.89$ & $34.31 \pm 6.50$ & $<0.001^{*}$ \\
\hline Waist circumference $(\mathrm{cm})$ & $90.69 \pm 9.27$ & $82.84 \pm 7.16$ & $94.30 \pm 7.79$ & $<0.001^{*}$ \\
\hline Systolic blood pressure (mmHg) & $142.470 \pm 19.77$ & $129.83 \pm 14.75$ & $148.28 \pm 19.10$ & $<0.001^{*}$ \\
\hline Diastolic blood pressure (mmHg) & $82.77 \pm 11.07$ & $76.85 \pm 9.02$ & $80.50 \pm 10.89$ & $<0.001^{*}$ \\
\hline Albumin $(\mathrm{mg} / \mathrm{dL})$ & $4.28 \pm 0.27$ & $4.27 \pm 0.20$ & $4.29 \pm 0.29$ & 0.565 \\
\hline Total cholesterol (mg/dL) & $162.25 \pm 30.69$ & $161.962 \pm 29.54$ & $162.39 \pm 31.33$ & 0.934 \\
\hline Triglyceride (mg/dL) & $115.00(85.00-172.00)$ & $92.50(61.25-125.75)$ & $127.00(93.50-192.00)$ & $<0.001^{*}$ \\
\hline HDL-C (mg/dL) & $46.69 \pm 12.58$ & $51.92 \pm 14.47$ & $44.28 \pm 10.86$ & $<0.001^{*}$ \\
\hline LDL-C (mg/dL) & $99.92 \pm 26.58$ & $97.40 \pm 23.85$ & $101.08 \pm 27.77$ & 0.411 \\
\hline Fasting glucose (mg/dL) & $138.00(121.00-175.00)$ & $125.50(116.250-157.75)$ & $143.00(126.00-179.50)$ & $0.033^{*}$ \\
\hline Glycated hemoglobin (\%) & $7.50(6.60-9.00)$ & $7.00(6.25-8.10)$ & $7.90(6.70-9.30)$ & $0.007^{*}$ \\
\hline Blood urea nitrogen $(\mathrm{mg} / \mathrm{dL})$ & $16.00(12.00-18.50)$ & $15.00(12.00-17.00)$ & $16.00(13.005-19.50)$ & $0.020^{*}$ \\
\hline Creatinine $(\mathrm{mg} / \mathrm{dL})$ & $0.90(0.70-1.00)$ & $0.90(0.70-1.00)$ & $0.80(0.70-1.00)$ & 0.741 \\
\hline Glomerular filtration rate $(\mathrm{mL} / \mathrm{min})$ & $87.23 \pm 27.80$ & $74.70 \pm 21.15$ & $65.96 \pm 20.31$ & $0.021^{*}$ \\
\hline hs-CRP (mg/dL) & $0.08(0.05-0.25)$ & $0.05(0.05-0.11)$ & $0.11(0.05-0.34)$ & $<0.001$ \\
\hline Insulin $(\mu \mathrm{IU} / \mathrm{mL})$ & $6.93(3.62-14.64)$ & $3.74(2.10-6.10)$ & $9.63(5.27-17.68)$ & $<0.001^{*}$ \\
\hline HOMA-IR & $2.47(1.24-5.08)$ & $1.26(0.77-2.14)$ & $3.55(1.91-6.93)$ & $<0.001^{*}$ \\
\hline A-FABP (ng/mL) & $28.49 \pm 15.55$ & $20.05 \pm 9.91$ & $32.37 \pm 16.16$ & $<0.001^{*}$ \\
\hline Female (\%) & $73(44.2)$ & $14(26.9)$ & $59(52.2)$ & $0.002^{*}$ \\
\hline Hypertension (\%) & $86(52.1)$ & $17(32.7)$ & $69(61.1)$ & $0.001^{*}$ \\
\hline Smoking (\%) & $17(10.3)$ & $6(11.5)$ & $11(9.7)$ & 0.723 \\
\hline
\end{tabular}

Values for continuous variables are given as means \pm standard deviation and are tested by Student's $t$-test; variables not normally distributed are given as medians and interquartile range and are tested by Mann-Whitney $U$ test; values are presented as number (\%) and analysis was done using the chi-square test. HDL-C, high-density lipoprotein cholesterol; LDL-C, low-density lipoprotein cholesterol; hs-CRP, high-sensitivity C-reactive protein; HOMA-IR, homeostasis model assessment of insulin resistance; A-FABP, adipocyte fatty acid binding protein.

${ }^{*} P<0.05$ was considered statistically significant after Student's $t$-test or Mann-Whitney $U$ test.

morning using standard mercury sphygmomanometers with appropriate cuff sizes after sitting for at least 10 minutes. Systolic BP (SBP) and diastolic BP (DBP) were taken 3 times at $5 \mathrm{~min}$ intervals and were averaged for analysis. Patients who were diagnosed with hypertension were defined as SBP $\geq$ $140 \mathrm{mmHg}$ and/or DBP $\geq 90 \mathrm{mmHg}$ or have received any antihypertensive medication in the past 2 weeks. Patients were excluded if they had an acute infection, acute myocardial infarction, heart failure, and malignancy at the time of blood sampling, or if they refused to provide informed consent for the study.

2.2. Anthropometric Analysis. Body weight of participant was measured in light clothing and without shoes to the nearest 0.5 kilograms, and body height was measured to the nearest $0.5 \mathrm{~cm}$. Waist circumference was measured using a tape measurement around the waist from the point between the lowest ribs and the hip bones with the hands on the hips. Body mass index (BMI) was calculated as the weight in kilograms divided by the height in meters squared. Bioimpedance measurements of fat mass were performed at the bedside according to the standard tetrapolar whole body (handfoot) technique, using a single-frequency $(50 \mathrm{kHz})$ analyzer (Biodynamic-450, Biodynamics Corporation, Seattle, USA). Measurements were carried out by the same operator [6-8].

2.3. Biochemical Investigations. Fasting blood samples (approximately $5 \mathrm{~mL}$ ) of all participants were immediately centrifuged at $3000 \times \mathrm{g}$ for $10 \mathrm{~min}$. Serum levels of albumin, blood urea nitrogen (BUN), creatinine, fasting glucose, glycated hemoglobin (HbAlc), total cholesterol (TCH), triglycerides (TG), high-density lipoprotein cholesterol (HDL-C), lowdensity lipoprotein cholesterol (LDL-C), and high-sensitivity C-reactive protein (hs-CRP) were measured using an autoanalyzer (Siemens Advia 1800, Siemens Healthcare GmbH, Henkestr, Germany) [6-8]. Serum A-FABP levels were measured using a commercially available enzyme immunoassay (EIA; SPI-BIO, Montigny le Bretonneux, France) [6-8]. 
TABLE 2: Clinical characteristics and fasting serum adipocyte fatty acid binding protein levels of 165 diabetic patients.

\begin{tabular}{lccc}
\hline Characteristic & & Number $(\%)$ & A-FABP $(\mathrm{ng} / \mathrm{mL})$ \\
\hline \multirow{2}{*}{ Gender } & Male & $92(55.7)$ & $23.90 \pm 12.44$ \\
& Female & $73(44.3)$ & $34.27 \pm 17.16$ \\
Hypertension & No & $79(47.9)$ & $26.16 \pm 15.49$ \\
& Yes & $86(52.1)$ & $30.63 \pm 15.38$ \\
Smoking & No & $148(89.7)$ & $28.54 \pm 15.55$ \\
& Yes & $17(10.3)$ & $28.07 \pm 16.03$ \\
Statin & No & $84(50.9)$ & $26.83 \pm 13.07$ \\
& Yes & $81(49.1)$ & $30.21 \pm 17.68$ \\
Fibrate & No & $156(94.5)$ & $28.14 \pm 15.73$ \\
& Yes & $9(5.5)$ & $34.57 \pm 10.78$ \\
Metformin & No & $74(44.8)$ & $28.47 \pm 16.54$ \\
& Yes & $91(55.2)$ & $28.51 \pm 14.79$ \\
Sulfonylureas & No & $77(46.7)$ & $29.07 \pm 16.50$ \\
& Yes & $88(53.3)$ & $27.98 \pm 14.75$ \\
DDP-4 inhibitor & No & $65(39.4)$ & $28.03 \pm 17.09$ \\
& Yes & $100(60.6)$ & $28.79 \pm 14.54$ \\
Thiazolidinediones & No & $160(97.0)$ & $28.26 \pm 14.60$ \\
Insulin & Yes & $5(3.0)$ & $35.88 \pm 37.05$ \\
& No & $122(73.9)$ & $28.39 \pm 15.68$
\end{tabular}

Data are expressed as means \pm standard deviations.

${ }^{*} P<0.05$ was considered statistically significant after Student's $t$-test.

DDP-4, dipeptidyl peptidase 4 .

Serum insulin levels were measured using the commercially available enzyme-linked immunosorbent assay (ELISA) (Labor Diagnostika Nord, Nordhorn, Germany) $[9,10]$. Insulin resistance was evaluated using a homeostasis model assessment of insulin resistance (HOMA-IR) as follows: HOMA$\mathrm{IR}=$ fasting plasma glucose $(\mathrm{mg} / \mathrm{dL}) \times$ fasting serum insulin $(\mu \mathrm{U} / \mathrm{mL}) / 405[9,10]$. The estimate glomerular filtration rate (GFR) was calculated using the Modification of Diet in Renal Disease (MDRD) study equation in this study.

2.4. Metabolic Syndrome and Its Components. The prevalence of MetS was defined using the International Diabetes Federation definition [11]. People were classified as having MetS if they had central (abdominal) obesity with a waist circumference $\geq 90 \mathrm{~cm}$ (men) or $\geq 80 \mathrm{~cm}$ (women) (Chinese criteria) and matched two or more of the following criteria: fasting serum glucose $\geq 110 \mathrm{mg} / \mathrm{dL}$, triglycerides $\geq 150 \mathrm{mg} / \mathrm{dL}$, HDL-C level $<40 \mathrm{mg} / \mathrm{dL}$ in men or $<50 \mathrm{mg} / \mathrm{dL}$ in women, or blood pressure $\geq 130 / 85 \mathrm{mmHg}$. The use of antihypertensive drug was considered as indicative of high blood pressure in this analysis. Type $2 \mathrm{DM}$ was determined according to World Health Organization criteria [12]. A patient was considered DM patient if the fasting plasma glucose was $\geq 126 \mathrm{mg} / \mathrm{dL}$, or if antidiabetic therapy was used [6-8].

2.5. Statistical Analysis. Data were tested for normal distribution using the Kolmogorov-Smirnov test. Normally distributed data are expressed as the mean \pm standard deviation (SD) and comparisons between patients were performed using Student's independent $t$-test (two-tailed). Data are not normally distributed are expressed as medians and interquartile ranges and comparisons between patients were performed using the Mann-Whitney $U$ test (age, TG, fasting glucose, HbAlc, BUN, creatinine, hs-CRP, insulin, and HOMA-IR). Data expressed as the number of patients were analyzed by $\chi^{2}$ test, because age, TG, fasting glucose, HbAlc, BUN, creatinine, hs-CRP, insulin, and HOMA-IR were not normally distributed and underwent base 10 logarithmic transformations to achieve normality. Clinical variables that correlated with serum A-FABP levels in DM patients were evaluated using univariate linear regression analysis. Variables that were significantly associated with A-FABP levels in DM patients were tested for independency in multivariate forward stepwise regression analysis. Data were analyzed using SPSS for Windows (version 19.0; SPSS Inc., Chicago, IL, USA). A $P$ value $<0.05$ was considered statistically significant.

\section{Results}

Clinical characteristics of the 165 type 2 DM patients are presented in Table 1. One hundred thirteen DM persons (68.5\%) had MetS, and 52 DM persons (31.5\%) did not. Diabetic persons who had MetS had significantly higher serum fasting A-FABP levels than those without MetS $(P<$ 0.001). Compared with DM patients without MetS, those with MetS showed a much higher proportion of females $(P=$ $0.002)$ and as expected more hypertension $(P=0.001)$, 
TABLE 3: Correlation of fasting serum adipocyte fatty acid binding protein levels and clinical variables by univariable linear regression analyses among the 165 diabetic patients.

\begin{tabular}{lcc}
\hline Items & Beta & $P$ value \\
\hline Log-age (years) & 0.115 & 0.140 \\
Height $(\mathrm{cm})$ & -0.293 & $<0.001^{*}$ \\
Body weight $(\mathrm{kg})$ & -0.031 & 0.696 \\
Body mass index $\left(\mathrm{kg} / \mathrm{m}^{2}\right)$ & 0.173 & $0.026^{*}$ \\
Body fat mass $(\%)$ & 0.394 & $<0.001^{*}$ \\
Waist circumference $(\mathrm{cm})$ & 0.166 & $0.033^{*}$ \\
Systolic blood pressure $(\mathrm{mmHg})$ & 0.324 & $<0.001^{*}$ \\
Diastolic blood pressure $(\mathrm{mmHg})$ & 0.173 & $0.026^{*}$ \\
Albumin $(\mathrm{mg} / \mathrm{dL})$ & -0.259 & $0.001^{*}$ \\
Total cholesterol (mg/dL) & 0.179 & $0.022^{*}$ \\
Log-triglyceride $(\mathrm{mg} / \mathrm{dL})$ & 0.227 & $0.003^{*}$ \\
HDL-C (mg/dL) & -0.041 & 0.600 \\
LDL-C (mg/dL) & 0.099 & 0.204 \\
Log-glucose $(\mathrm{mg} / \mathrm{dL})$ & 0.133 & 0.088 \\
Log-HbAlc $(\%)$ & 0.225 & $0.004^{*}$ \\
Log-BUN (mg/dL) & 0.363 & $<0.001^{*}$ \\
Log-creatinine $(\mathrm{mg} / \mathrm{dL})$ & 0.257 & $0.001^{*}$ \\
Glomerular filtration rate $(\mathrm{mL} / \mathrm{min})$ & -0.343 & $<0.001^{*}$ \\
Log-hs-CRP $(\mathrm{mg} / \mathrm{dL})$ & 0.212 & $0.006^{*}$ \\
Log-insulin $(\mu \mathrm{IU} / \mathrm{mL})$ & 0.214 & $0.006^{*}$ \\
Log-HOMA-IR & 0.218 & $0.005^{*}$ \\
\hline Daf & &
\end{tabular}

Data of age, triglyceride, glucose, HbAlc, BUN, creatinine, and hs-CRP levels showed skewed distribution and therefore were log-transformed before analysis.

${ }^{*} P<0.05$ was considered statistically significant after univariable linear analyses.

Abbreviations: HDL-cholesterol, high-density lipoprotein cholesterol; LDLcholesterol, low-density lipoprotein cholesterol; BUN, blood urea nitrogen; hs-CRP, high-sensitivity C-reactive protein; HOMA-IR, homeostasis model assessment of insulin resistance.

higher waist circumference $(P<0.001)$, body weight $(P<$ $0.001)$, and BMI $(P<0.001)$, higher fasting glucoses $(P=$ $0.033)$, TG $(P=0.001)$, and lower HDL-C concentrations $(P<0.001)$ since MetS represents a constellation of hypertension, abdominal obesity, impaired fasting glucose, and dyslipidemia. Moreover, DM patients with MetS had higher body fat mass $(P<0.001)$, HbAlc level $(P=0.007)$, BUN $(P=0.020)$, hs-CRP $(P<0.001)$, insulin level $(P<0.001)$, HOMA-IR $(P<0.001)$, and lower GFR $(P=0.021)$.

Clinical characteristics and serum A-FABP values for the 165 DM patients are presented in Table 2. A-FABP level was statistically significantly higher in female DM persons than male DM persons $(P<0.001)$. No statistically significant differences in A-FABP levels were found as a function of smoking; presence of hypertension; or use of statin, fibrate, or antidiabetic drugs.

Univariate linear analysis of clinical variables associated with fasting serum A-FABP levels in DM patients is presented in Table 3. Height $(r=-0.293 ; P<0.001)$ and serum albumin level $(r=-0.259 ; P=0.001)$ were negatively correlated, while BMI $(r=0.173 ; P=0.026)$, body fat mass $(r=0.394 ; P<0.001), \operatorname{SBP}(r=0.324 ; P<0.001), \mathrm{DBP}$ $(r=0.173 ; P=0.026)$, TCH $(r=0.179 ; P=0.022)$, and logarithmically transformed TG (log-TG, $r=0.227$; $P=0.003$ ) were positively correlated with serum A-FABP levels in type 2 DM patients. Furthermore, log-HbAlc $(r=$ $0.225 ; P=0.004)$, log-BUN $(r=0.363 ; P<0.001)$, logcreatinine $(r=0.257 ; P=0.001)$, log-hs-CRP $(r=0.212$; $P=0.006)$, log-insulin $(r=0.214 ; P=0.006)$, and logHOMA-IR ( $r=0.218 ; P=0.005)$ were positively correlated, while GFR $(r=-0.343 ; P<0.001)$ was negatively correlated with serum A-FABP levels in this study.

Multivariate forward stepwise linear regression analysis of the variables significantly associated with fasting serum AFABP levels revealed that body fat mass (adjusted $R^{2}$ change $=$ $0.150 ; P<0.001$ ), log-creatinine (adjusted $R^{2}$ change $=0.140$; $P<0.001$ ), female DM patients (adjusted $R^{2}$ change $=0.051$; $P<0.001$ ), serum albumin level (adjusted $R^{2}$ change $=0.029$; $P=0.004$ ), log-hs-CRP (adjusted $R^{2}$ change $=0.020 ; P=$ 0.013 ), and GFR (adjusted $R^{2}$ change $=0.012 ; P=0.043$ ) were independent predictors of these values for type $2 \mathrm{DM}$ patients (Table 4).

\section{Discussion}

Our study showed that fasting A-FABP levels were higher in type $2 \mathrm{DM}$ patents with MetS, and body fat mass, creatinine, female, albumin, hs-CRP, and GFR were independent predictors of serum A-FABP levels in type $2 \mathrm{DM}$ patients.

MetS is defined by the presence of central obesity, hyperglycemia, type $2 \mathrm{DM}$, hypertension, and dyslipidemia [11]. The prevalence of MetS was $46.9 \%$ for males and $65.1 \%$ for females in type $2 \mathrm{DM}$ patients in a Korean study [13] and $78.6 \%$ in type $2 \mathrm{DM}$ patients in Brazil [14]. In this study, the prevalence of MetS in type $2 \mathrm{DM}$ patients is $68.5 \%$. MetS is strongly associated with insulin resistance and inflammation [11]. Patients with type $2 \mathrm{DM}$ and MetS showed significantly higher blood CRP and insulin resistance than type $2 \mathrm{DM}$ without MetS [13]. Our study noted that diabetic persons who had MetS had significantly higher in-body fat mass, HbAlc level, hs-CRP, insulin level, and HOMA-IR than those diabetic persons without MetS. Females are at greater risk of obesity and central adiposity due to their increased propensity to gain fat [15]. Our results found that diabetic persons who had MetS had higher trend in female gender. MetS and its components are associated with the development of chronic kidney disease and microalbuminuria or overt proteinuria [16]. Type $2 \mathrm{DM}$ patients with MetS are associated with the reduction of GFR [14]. Our study also noted that MetS in patients with type 2 DM had higher serum BUN level and lower GFR.

Studies in A-FABP knockout mice and A-FABP inhibitor treated animals suggest that A-FABP has an important role in lipolysis and regulating insulin sensitivity $[3,5,17,18]$. Recent studies also demonstrated A-FABP expression in macrophages and modulate inflammatory responses and cholesterol ester accumulation [3, 5, 19]. All of these results indicate that A-FABP has an important role in the development of major components of the MetS through its distinct 
TABLE 4: Multivariable stepwise linear regression analysis of gender, height, body mass index, waist circumference, body fat mass, systolic blood pressure, diastolic blood pressure, albumin, total cholesterol, log-triglyceride, log-HbAlc, log-BUN, log-creatinine, glomerular filtration rate, log-CRP, log-insulin, and log-HOMA-IR: correlation to fasting serum adipocyte fatty acid binding protein level among 165 diabetic patients.

\begin{tabular}{|c|c|c|c|c|}
\hline Items & Beta & Adjusted $R^{2}$ & Adjusted $R^{2}$ change & $P$ value \\
\hline Body fat mass (\%) & 0.211 & 0.150 & 0.150 & $<0.001^{*}$ \\
\hline Log-creatinine (mg/dL) & 0.843 & 0.290 & 0.140 & $<0.001^{*}$ \\
\hline Female & 0.513 & 0.341 & 0.051 & $<0.001^{*}$ \\
\hline Albumin (mg/dL) & -0.180 & 0.370 & 0.029 & $0.004^{*}$ \\
\hline Log-hs-CRP (mg/dL) & 0.154 & 0.390 & 0.020 & $0.013^{*}$ \\
\hline Glomerular filtration rate $(\mathrm{mL} / \mathrm{min})$ & -0.366 & 0.402 & 0.012 & $0.043^{*}$ \\
\hline
\end{tabular}

${ }^{*} P<0.05$ was considered statistically significant after multivariable stepwise linear regression analyses.

hs-CRP, high-sensitivity C-reactive protein; HOMA-IR, homeostasis model assessment of insulin resistance.

actions in adipocytes and macrophages. Serum A-FABP levels predict the development of the MetS in a Chinese and a Korean cohort study [20, 21] and plasma A-FABP level was found to be a strong predictor of type $2 \mathrm{DM}$ independently of the traditional risk factors including obesity, insulin resistance, or glycemic indexes [22]. Cabré et al. noted A-FABP levels positively correlated to the MetS in type 2 DM subjects [23]. Our study also noted higher serum AFABP level in type 2 DM patients who had MetS. Hao et al. noted serum A-FABP levels significantly higher in women than men [24]. Our study also noted that female diabetic patients had higher A-FABP levels than man. In type $2 \mathrm{DM}$ patients, A-FABP levels were positively correlated with BMI, TG, TCH, SBP, waist circumference, IL- 6 , TNF- $\alpha$, and hsCRP $[23,25]$. Furthermore, A-FABP levels were positively correlated with plasma TG, apolipoprotein C-III, and all the components of TG-rich lipoproteins in type 2 diabetic subjects [26]. A-FABP levels positively correlated with BMI, glucose, insulin levels, HOMA-IR, and CRP in morbidly obese women [27]. Subjects in the highest A-FABP tertile at baseline exhibited higher values for BMI, body fat mass, blood pressure, fasting glucose, TCH, TG, LDL-C, insulin, and HOMA-IR in a total of 465 participants in a Korean study [21]. A-FABP was independently associated with body fat mass in a cohort of Chinese women without DM [28]. Serum A-FABP concentrations were positively associated with serum creatinine and albumin and predict a worse hospital outcome in critically ill patients with sepsis [29]. Since serum creatinine is a component of the GFR estimation. Studies also noted serum A-FABP4 levels correlated positively with serum creatinine and negatively with GFR in type 2 DM patients $[30,31]$. Serum A-FABP level was independently associated with macrovascular complications in type $2 \mathrm{DM}$ patients [31]. We also noted that BMI, body fat mass, SBP, DBP, TCH, log-TG, log-creatinine, log-HbAlc, log-hs-CRP, log-insulin, and log-HOMA-IR were positively correlated with A-FABP levels, while height, albumin, and GFR were negatively correlated with A-FABP levels in our DM subjects. After adjustment for a variety of confounders in multivariable forward stepwise linear regression analysis, body fat mass, log-creatinine, female DM patients, and loghs-CRP remained to be positively associated, while serum albumin level and GFR negatively associated with A-FABP level.

Our study had some limitations. First, this study had a cross-sectional design without a control group with a limited number of participants enrolled and the possibility of bias cannot be excluded. Second, pharmacological interventions have been shown to influence serum A-FABP in humans. Thiazolidinedione increases plasma A-FABP concentrations in type $2 \mathrm{DM}$ subjects [23]. Another study noted that canagliflozin increases serum A-FABP levels in diabetic patients [32]. Our patients did not use any the sodium/glucose cotransporter 2 (SGLT2) inhibitor. Our results did not show a relationship between thiazolidinedione, stains, fibrates or other antidiabetic drugs, and serum A-FABP in the diabetic patients studied. Further studies are required to elucidate the relationship between medication and A-FABP in type $2 \mathrm{DM}$ patients.

\section{Conclusion}

In summary, the present study showed that serum A-FABP level was positively associated with MetS in the type $2 \mathrm{DM}$ patients. In addition, body fat mass, log-creatinine, female DM patients, and log-hs-CRP are positively correlated, while serum albumin and GFR are negatively correlated with AFABP level.

\section{Competing Interests}

The authors declare that they have no competing interests.

\section{Authors' Contributions}

Jer-Chuan $\mathrm{Li}$ and $\mathrm{Du}-\mathrm{An} \mathrm{Wu}$ contributed equally to this study.

\section{References}

[1] S. M. Grundy, "Metabolic syndrome update," Trends in Cardiovascular Medicine, vol. 26, no. 4, pp. 364-373, 2015.

[2] S. Gothai, P. Ganesan, S. Park, S. Fakurazi, D. Choi, and P. Arulselvan, "Natural phyto-bioactive compounds for the treatment 
of type 2 diabetes: inflammation as a target," Nutrients, vol. 8 , no. 8, p. E461, 2016.

[3] S. Kralisch and M. Fasshauer, "Adipocyte fatty acid binding protein: a novel adipokine involved in the pathogenesis of metabolic and vascular disease?" Diabetologia, vol. 56, no. 1, pp. 10-21, 2013.

[4] M. Furuhashi, M. Furuhashi, S. Saitoh, K. Shimamoto, and T. Miura, "Fatty acid-binding protein 4 (FABP4): pathophysiological insights and potent clinical biomarker of metabolic and cardiovascular diseases," Clinical Medicine Insights: Cardiology, vol. 8, supplement 3, pp. 23-33, 2015.

[5] A. Xu and P. M. Vanhoutte, "Adiponectin and adipocyte fatty acid binding protein in the pathogenesis of cardiovascular disease," American Journal of Physiology-Heart and Circulatory Physiology, vol. 302, no. 6, pp. H1231-H1240, 2012.

[6] J.-P. Tsai, H.-H. Liou, H.-M. Liu, C.-J. Lee, R.-P. Lee, and B.-G. $\mathrm{Hsu}$, "Fasting serum fatty acid-binding protein 4 level positively correlates with metabolic syndrome in hemodialysis patients," Archives of Medical Research, vol. 41, no. 7, pp. 536-540, 2010.

[7] B.-G. Hsu, Y.-C. Chen, R.-P. Lee, C.-C. Lee, C.-J. Lee, and J.H. Wang, "Fasting serum level of fatty-acid-binding protein 4 positively correlates with metabolic syndrome in patients with coronary artery disease," Circulation Journal, vol. 74, no. 2, pp. 327-331, 2010.

[8] J.-P. Tsai, J.-H. Wang, C.-J. Lee, Y.-C. Chen, and B.-G. Hsu, "Positive correlation of serum adipocyte fatty acid binding protein levels with carotid-femoral pulse wave velocity in geriatric population," BMC Geriatrics, vol. 15, article 88, 2015.

[9] J.-H. Wang, C.-J. Lee, J.-C. Hsieh, Y.-C. Chen, and B.-G. $\mathrm{Hsu}$, "Inverse association of long-acting natriuretic peptide with metabolic syndrome in congestive heart failure patients," Diabetology and Metabolic Syndrome, vol. 5, article 19, 2013.

[10] J.-C. Hsieh, J.-H. Wang, C.-J. Lee, Y.-C. Chen, H.-H. Liou, and B.-G. Hsu, "Low serum long-acting natriuretic peptide level correlates with metabolic syndrome in hypertensive patients: a cross-sectional study," Archives of Medical Research, vol. 44, no. 3, pp. 215-220, 2013.

[11] K. G. M. M. Alberti, P. Z. Zimmet, and J. Shaw, "Metabolic syndrome-a new world-wide definition. A consensus statement from the International Diabetes Federation," Diabetic Medicine, vol. 23, no. 5, pp. 469-480, 2006.

[12] K. G. Alberti and P. Z. Zimmet, "Definition, diagnosis and classification of diabetes mellitus and its complications. Part 1: diagnosis and classification of diabetes mellitus provisional report of a WHO consultation," Diabetic Medicine, vol. 15, no. 7, pp. 539-553, 1998.

[13] W. Y. Kim, J. E. Kim, Y. J. Choi, and K. B. Huh, "Nutritional risk and metabolic syndrome in Korean type 2 diabetes mellitus," Asia Pacific Journal of Clinical Nutrition, vol. 17, supplement 1, pp. 47-51, 2008.

[14] M. Moehlecke, C. B. Leitão, C. K. Kramer et al., "Effect of metabolic syndrome and of its individual components on renal function of patients with type 2 diabetes mellitus," Brazilian Journal of Medical and Biological Research, vol. 43, no. 7, pp. 687693, 2010.

[15] F. Mauvais-Jarvis, "Sex differences in metabolic homeostasis, diabetes, and obesity," Biology of Sex Differences, vol. 6, article $14,2015$.

[16] G. Thomas, A. R. Sehgal, S. R. Kashyap, T. R. Srinivas, J. P. Kirwan, and S. D. Navaneethan, "Metabolic syndrome and kidney disease: a systematic review and meta-analysis," Clinical
Journal of the American Society of Nephrology, vol. 6, no. 10, pp. 2364-2373, 2011.

[17] N. R. Coe, M. A. Simpson, and D. A. Bernlohr, “Targeted disruption of the adipocyte lipid-binding protein (aP2 protein) gene impairs fat cell lipolysis and increases cellular fatty acid levels," Journal of Lipid Research, vol. 40, no. 5, pp. 967-972, 1999.

[18] L. Scheja, L. Makowski, K. T. Uysal et al., "Altered insulin secretion associated with reduced lipolytic efficiency in aP2-/mice," Diabetes, vol. 48, no. 10, pp. 1987-1994, 1999.

[19] L. Makowski, J. B. Boord, K. Maeda et al., "Lack of macrophage fatty-acid-binding protein aP2 protects mice deficient in apolipoprotein E against atherosclerosis," Nature Medicine, vol. 7, no. 6, pp. 699-705, 2001.

[20] A. Xu, A. W. K. Tso, B. M. Y. Cheung et al., "Circulating adipocyte-fatty acid binding protein levels predict the development of the metabolic syndrome: a 5-year prospective study," Circulation, vol. 115, no. 12, pp. 1537-1543, 2007.

[21] S. E. Park, E.-J. Rhee, W.-Y. Lee et al., “The role of serum adipocyte fatty acid-binding protein on the development of metabolic syndrome is independent of pro-inflammatory cytokines," Nutrition, Metabolism and Cardiovascular Diseases, vol. 22, no. 6, pp. 525-532, 2012.

[22] A. W. Tso, A. Xu, P. C. Sham et al., "Serum a new biomarker predicting the development of a 10-year prospective study in a," Diabetes Care, vol. 30, no. 10, pp. 2667-2672, 2007.

[23] A. Cabré, I. Lázaro, J. Girona et al., "Fatty acid binding protein 4 is increased in metabolic syndrome and with thiazolidinedione treatment in diabetic patients," Atherosclerosis, vol. 195, no. 1, pp. e150-e158, 2007.

[24] Y. Hao, X. Ma, Y. Luo et al., "Serum adipocyte fatty acid binding protein levels are positively associated with subclinical atherosclerosis in chinese pre- and postmenopausal women with normal glucose tolerance," Journal of Clinical Endocrinology and Metabolism, vol. 99, no. 11, pp. 4321-4327, 2014.

[25] G. Aragonès, R. Ferré, I. Lázaro et al., "Fatty acid-binding protein 4 is associated with endothelial dysfunction in patients with type 2 diabetes," Atherosclerosis, vol. 213, no. 1, pp. 329-331, 2010.

[26] A. Cabré, I. Lázaro, J. Girona et al., "Plasma fatty acid binding protein 4 is associated with atherogenic dyslipidemia in diabetes," Journal of Lipid Research, vol. 49, no. 8, pp. 1746-1751, 2008.

[27] X. Terra, Y. Quintero, T. Auguet et al., "FABP 4 is associated with inflammatory markers and metabolic syndrome in morbidly obese women," European Journal of Endocrinology, vol. 164, no. 4, pp. 539-547, 2011.

[28] Y. Hao, X. Ma, Y. Luo et al., "Associations of serum adipocyte fatty acid binding protein with body composition and fat distribution in nondiabetic Chinese women," The Journal of Clinical Endocrinology and Metabolism, vol. 100, no. 5, pp. 20552062, 2015.

[29] C.-L. Huang, Y.-W. Wu, A.-R. Hsieh, Y.-H. Hung, W.-J. Chen, and W.-S. Yang, "Serum adipocyte fatty acid-binding protein levels in patients with critical illness are associated with insulin resistance and predict mortality," Critical Care, vol. 17, article R22, 2013.

[30] F. Toruner, A. E. Altinova, M. Akturk et al., "The relationship between adipocyte fatty acid binding protein-4, retinol binding protein-4 levels and early diabetic nephropathy in patients with type 2 diabetes," Diabetes Research and Clinical Practice, vol. 91, no. 2, pp. 203-207, 2011. 
[31] D. C. Y. Yeung, A. Xu, A. W. K. Tso et al., "Circulating levels of adipocyte and epidermal fatty acid-binding proteins in relation to nephropathy staging and macrovascular complications in type 2 diabetic patients," Diabetes Care, vol. 32, no. 1, pp. 132$134,2009$.

[32] M. Furuhashi, M. Matsumoto, S. Hiramitsu et al., "Possible increase in serum FABP4 level despite adiposity reduction by canagliflozin, an SGLT2 inhibitor," PLoS ONE, vol. 11, no. 4, Article ID e0154482, 2016. 


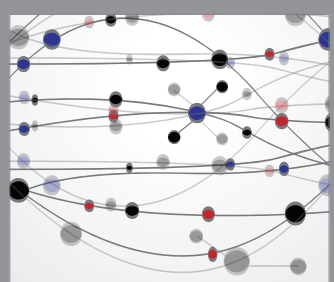

The Scientific World Journal
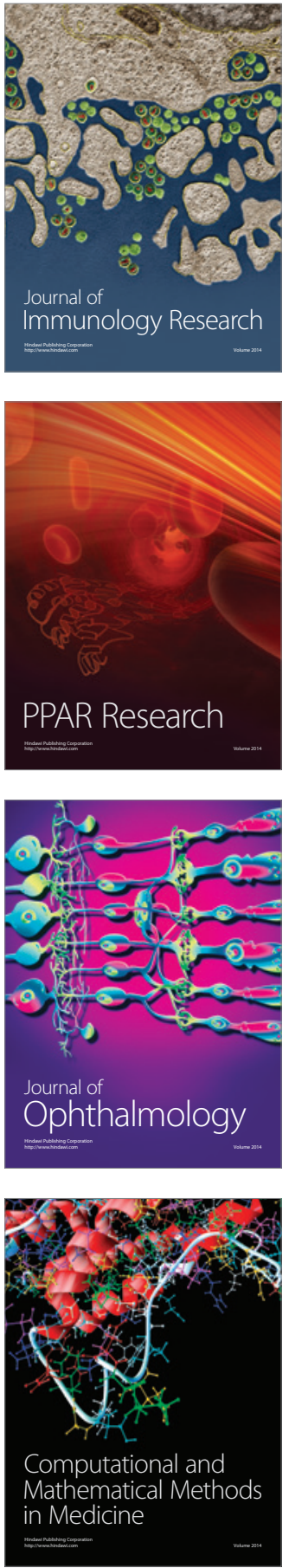

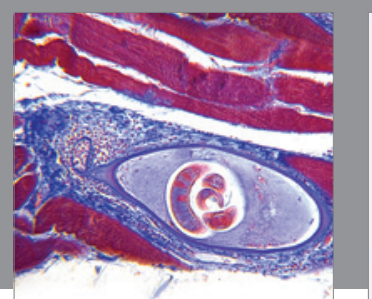

Gastroenterology Research and Practice

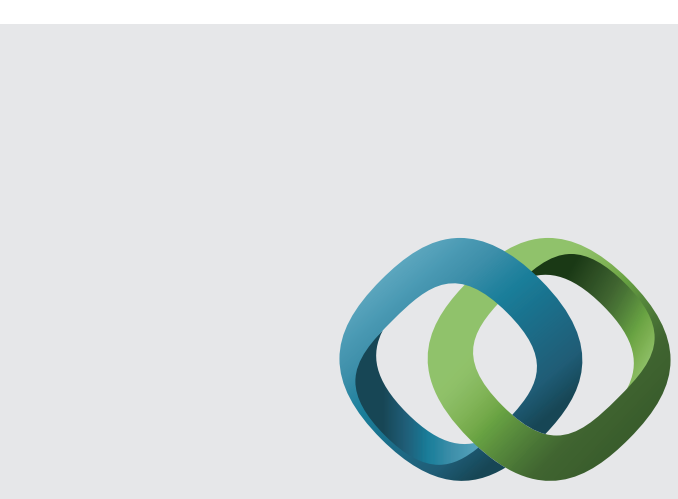

\section{Hindawi}

Submit your manuscripts at

http://www.hindawi.com
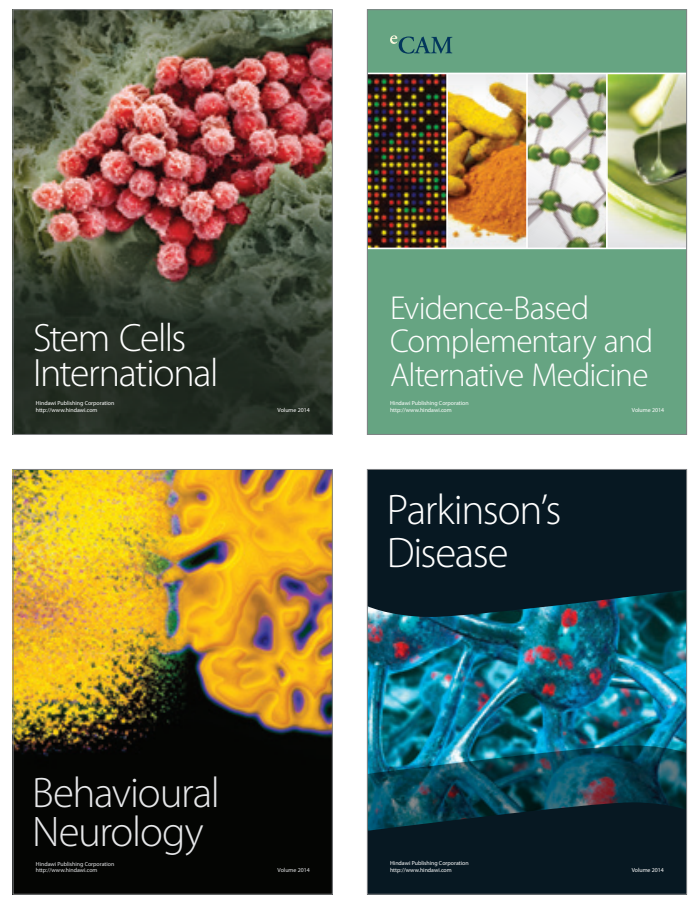
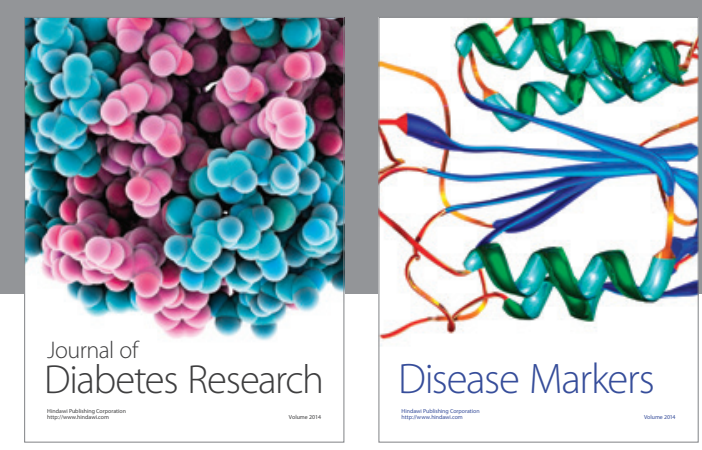

Disease Markers
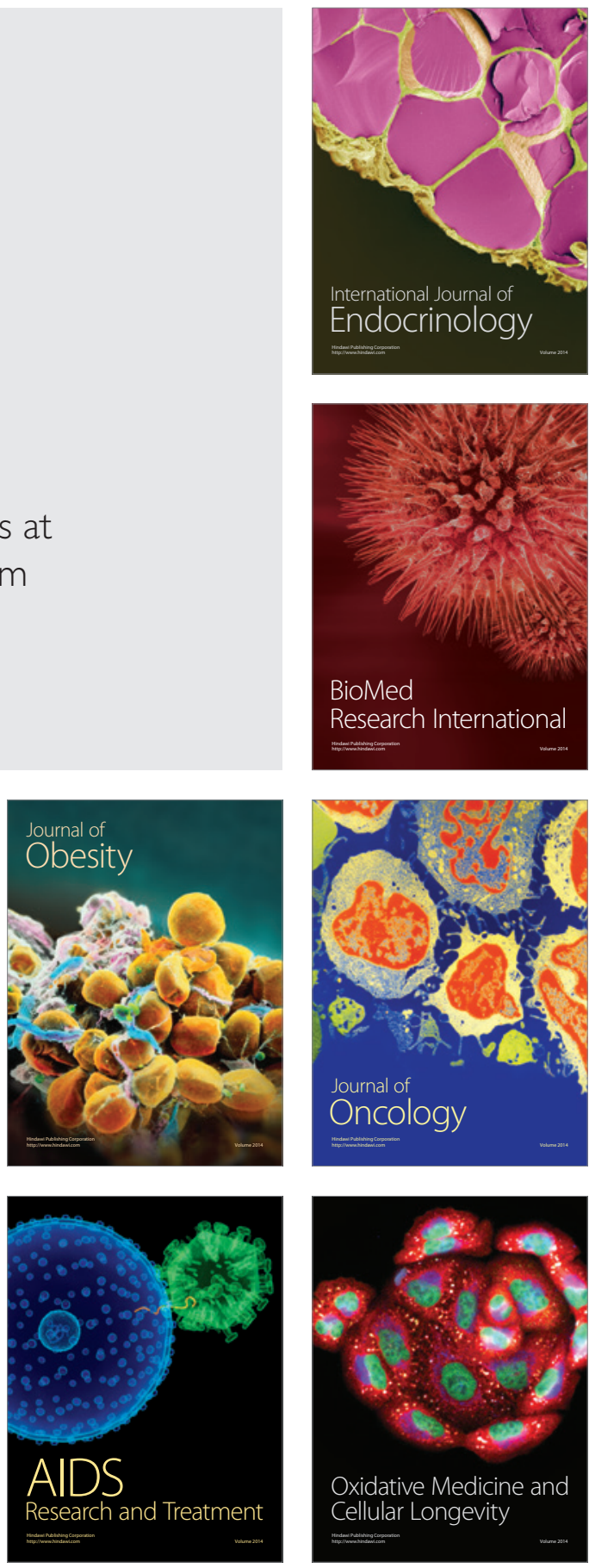